International

\title{
Oral Selenium: Can It Prevent Contrast-Induced Nephropathy Following Coronary Angiography and Angioplasty
}

\author{
Hashem Danesh Sani, ${ }_{1}^{1}$ Moosa Karami Kharat, ${ }_{1}^{1}$ Mohammad Vejdanparast, ${ }^{1}$ Ali Eshraghi, ${ }^{1,}$ Alireza \\ Abdollahi Moghaddam, ${ }^{1}$ and Hamid Eshraghi ${ }^{2}$
}

\footnotetext{
${ }^{1}$ Atherosclerosis Prevention Research Center, Imam Reza Hospital, Faculty of Medicine, Mashhad University of Medical Sciences, Mashhad, IR Iran ${ }^{2}$ Department of Internal Medicine, Qaem Hospital, Mashhad University of Medical Sciences, Mashhad, IR Iran
}

\begin{tabular}{l}
\hline A R T I C L E I N F O \\
\hline Article Type: \\
Research Article \\
\hline
\end{tabular}

Article History:

Received: 18 Jul 2014

Revised: 14 Mar 2015

Accepted: 08 Apr 2015

\section{Keywords:}

Nephropathy

Contrast Media

Angioplasty

Selenium

\begin{abstract}
A B S T R A C T
Background: Alteration of kidney function called Contrast-Induced Nephropathy (CIN) is commonly encountered in diagnostic and interventional cardiology procedures. It results in considerable morbidity and mortality besides imposing significant costs on the healthcare system. Today, it is accounted as the third cause of hospital-acquired acute renal failure.

Objectives: Considering anti-oxidant and free radical scavenging properties of selenium and the role of free radicals in CIN, the present study aimed to investigate the effects of oral selenium (in the form of Se-yeast capsule) on preventing or decreasing the rate of CIN in patients undergoing angiography and angioplasty.

Patients and Methods: This prospective, non-randomized, single-blind, single-center clinical trial was conducted on 175 consecutive patients admitted for elective coronary angiography $(\mathrm{N}=105)$ or angioplasty $(\mathrm{N}=70)$. Selenium was prescribed as a single dose of 200 micrograms the day before and after the procedure. Routine hydration based on the ward protocol was administered for all the patients. For the control group, the researchers made use of the data of another survey performed in the same hospitals for determining the prevalence of CIN a year before. The data were analyzed using student's t-test and logistic regression analysis and $\mathrm{P}<0.05$ was considered as statistically significant.

Results: Based on the results of student's t-test, the mean difference between the two groups was significant only in hematocrit levels. The incidence of CIN was $9 \%$ in the selenium group compared to $22 \%$ in the control group $(\mathrm{P}=0.027)$. There was no case of in-hospital mortality and none of the CIN patients required renal replacement therapy during hospitalization.
\end{abstract}

Conclusions: Prophylactic selenium administration may significantly reduce the incidence of CIN.

- Implication for health policy/practice/research/medical education:

This research article deals with the concept that life skills and the way an individual approaches them have a significant impact on one's health. Therefore, it is strongly recommended that health is related to psych.

\section{Background}

Alteration of kidney function called Contrast-Induced Nephropathy (CIN) is commonly encountered in diagnostic

*Corresponding author: Ali Eshraghi, Atherosclerosis Prevention Research Center, Imam Reza Hospital, Faculty of Medicine, Mashhad, Iran, Tel/Fax: +98-5118544504,

E-mail: alesh81036@yahoo.com and interventional cardiology procedures. It results in considerable morbidity and mortality besides imposing significant costs on the healthcare system. Acute renal failure due to contrast material exposure has been defined as absolute ( $\geq 0.5 \mathrm{mg} / \mathrm{dL})$ and/or $25 \%$ relative increase from baseline serum creatinine (1). Recent years have witnessed an increase in contrast media exposure, but today it is 
accounted as the third cause of hospital-acquired acute renal failure $(2,3)$. CIN occurs in more than $15 \%$ of patients undergoing cardiac catheterization (4). Also, its prevalence has been reported to be around $13 \%$ in non-diabetic cases and $20 \%$ in diabetics. In $1 \%$ of the cases, dialysis is required and in half of them, permanent dialysis will be the case. CIN is more common in heart failure, chronic renal failure, and diabetic patients. It is also associated with the volume of the used contrast media. Yet, the exact pathophysiological mechanism of CIN is not well understood. Alteration of renal hemodynamics or direct toxic effects of contrast media on tubular cells may be responsible (2). Enhanced formation of Reactive Oxygen Species (ROS) in kidney following contrast media exposure may also play a central role in this pathology (5). Hypoxia and formation of this reactive species may result in injury of tubular and parenchymal cells in kidney tissue. This may help explain the protective effect of free radical scavengers, such as $\mathrm{N}$-acetyl cysteine (6).

Regarding the rise in diagnostic and therapeutic cardiac procedures requiring contrast media, the incidence of CIN has also increased, which highlights the need for taking certain measures. At present, different methods are being developed to prevent CIN, including antioxidants agents, intrarenal vasodilators, adequate hydration, and use of less toxic iodine-containing contrast agents (7). One of the main measures is decreasing the amount of contrast media used to the lowest possible limit and preventing fluid loss in patients by starting hydration therapy before exposure to such substances. Another preventive approach is the use of substances that can lessen such complications. $\mathrm{N}$-acetyl cystein, sodium bicarbonate, Vitamin C, theophyline, fenoldopam, statins, and low dose dopamine and pentoxiphylline are examples of the substances used so far for this purpose, among which $\mathrm{N}$ - acetyl cystein has been more prominent $(2,8,9)$.

Considering the anti-oxidant and free radical scavenging properties of selenium $(10,11)$ and the role of free radicals in CIN, selenium supplement was proposed as a potential preventive measure in CIN.

\section{Objectives}

The present study aims to determine the protective role of selenium in reducing the incidence of CIN.

\section{Patients and Methods}

This prospective, non-randomized, single-blind, singlecenter, clinical trial was conducted on 203 consecutive patients admitted for elective coronary angiography or angioplasty in Qaem and Imam Reza hospitals, Mashhad, Iran. The study sample size was determined based on another study conducted on Pentoxiphylline (2). Selenium capsules containing 200 micrograms of selenium in the form of Se-yeast (21st Century ${ }^{\circledR}$ HealthCare, Inc. USA) were prescribed one day before the procedure and repeated as a single dose the day after the procedure. Routine hydration based on the ward protocol was administered for all the patients (100 cc per hour normal saline 6 hours before the procedure until 12 hours after that). The mean length of hospital stay was 1.3 days.
A year before, a survey was performed on the prevalence of CIN in the same hospitals. That study was conducted on 585 candidates of elective angiography or angioplasty. These patients had received only routine hydration with no other pharmaceutical drug for CIN prevention before the procedure. The data of these patients was used as the control group in our study. In that study, all the patients had undergone diagnostic angiography with or without left ventricular or aorta injection.

Serum creatinine level of both groups was measured before the procedure and 48 hours after that by a single laboratory staff who was blind to the study groups. Mehran algorithm was used for determining the predicted risk of CIN (12).

The inclusion criteria of the study were being above 18 years of age and having to undergo elective angiography or angioplasty. On the other hand, the exclusion criteria of the study were contraindication for normal saline solution, consumption of theophylin, Non-Steroidal Anti-Inflammatory Drugs (NSAIDS), aminoglycosides, and N-acetyl cystein, suffering from underlying renal failure with creatinine levels $\geq 1.5 \mathrm{mg} / \mathrm{dL}$, undergoing hemodialysis, having allergy to contrast media, having history of multiple myeloma, pulmonary edema, and uncontrolled hypertension, need for emergent angiography, diagnostic use of contrast media during the last two weeks, and having a history of congestive heart failure.

The study protocol was approved by the institutional Ethics Committee and written informed consents were obtained from all the patients. Coronary angioplasty was performed using the iso-osmolar nonionic contrast media Iodixanol (Visipaque 320, GE Healthcare, Cork, Ireland). The primary end-point of the study was occurrence of CIN, defined as a minimum $0.5 \mathrm{mg} / \mathrm{dL}$ or $25 \%$ increase in serum creatinine above the baseline 48 hours after exposure to the contrast media.

After all, the data were entered into the SPSS statistical software, version 13 (SPSS Inc, Chicago, IL, USA) and were analyzed using student's t-test. Moreover, all the variables; i.e, sex, age, hematocrite level, volume of the used contrast media, diabetes mellitus, hypertension, hyperlipidemia, and smoking, were entered into the regression analysis model and compared between the two groups to determine the independent role of each variable. Besides, $\mathrm{P}<0.05$ was considered as statistically significant.

\section{Results}

The present study was conducted on 813 patients. Femoral artery was the access site in all the patients. In the beginning, 15 patients were excluded because of $\mathrm{N}$-acetyl cystein consumption and 10 due to creatinine levels over $1.5 \mathrm{mg} /$ $\mathrm{dL}$. Among the remaining cases, 585 (74\%) received normal saline, whereas selenium was prescribed for the other 203 (26\%). In the study group, 23 patients were excluded because of refusal of creatinine level re-check after the procedure. Additionally, 5 patients were scheduled for emergent Coronary Artery Bypass Grafting (CABG) and were referred to the Cardiac Surgery Department. Due to the confounding effect of cardiopulmonary bypass on renal function, they were also excluded from the study. Finally, 
175 patients completed the study (105 patients undergoing angiography and 70 patients undergoing angioplasty).

Demographic and Para clinical data of the study and control groups have been presented in Table 1. Based on the results of student's t-test, the mean difference between the two groups was significant only in hematocrit levels.

The incidence of CIN was $9 \%$ in the selenium group compared to $22 \%$ in the control group $(\mathrm{P}=0.027)$. Sex, age, hematocrit level, volume of the used contrast media, diabetes mellitus, hypertension, hyperlipidemia, smoking, and selenium use were used in multivariate analysis model for predicting CIN. Multivariate analysis using logistic regression (backward step) showed that volume of the used contrast media $(\mathrm{P}=0.032, \mathrm{~B}=0.994)$ and selenium use $(\mathrm{P}=0.027, \mathrm{~B}=0.487)$ had significant effects on contrast nephropathy (Table 2). It should be noted that there was no case of in-hospital mortality and none of the CIN patients required renal replacement therapy during hospitalization.

\section{Discussion}

This non-randomized clinical trial aimed at investigating the effects of oral selenium (in the form of Se-yeast capsules) on preventing or decreasing the rate of CIN in patients undergoing angiography and angioplasty. CIN is still a noticeable problem in clinical practice. Although newer contrast agents are less nephrotoxic, the risk of CIN continues to be considerable and risk of morbidity and mortality increases in the affected patients. This may necessitate shortterm hemodialysis, which increases the length of hospital stay and risk of permanent renal damage (5).

Following exposure to contrast media, medullary blood flow and oxygenation declines and concentration of ROS, generated principally in outer medulla, may increase
(5). Generation of ROS is proportional to the amount of administered contrast media and altered antioxidant systems may further intensify the process of renal ROS formation (5). The evidence for increased ROS formation following contrast media exposure suggests the possibility that oxidative stress may play an important role in the pathogenesis of CIN (5).

In addition to adequate hydration before contrast exposure, pharmaceutical agents have been proposed for prevention of CIN. Most of these agents, such as $\mathrm{N}$-acetyl cysteine and ascorbic acid, have ROS scavenging properties. Pentoxiphylline is one of the newly introduced drugs for CIN prevention $(2,13,14)$. The first study on pentoxiphylline was conducted by Firouzi et al. (2), which was followed by the study by Yavari et al. (14). In these trials, the free radical scavenging property of pentoxiphylline was the basis for selection of this agent. The first study indicated the preventive effects of pentoxiphylline, whereas the latter demonstrated that pentoxphylline did not reduce the risk of CIN in patients with normal renal function undergoing Percutaneous Coronary Intervention (PCI).

Oral selenium is cheap, is readily available, and has no major cardiac complications or other side effects. Seleniumenriched yeast (Se-yeast) is a common form of selenium with relatively good bioavailability used to supplement the dietary intake of this important trace mineral (11) that reaches its peak serum concentration in about 9 hours after a single oral dose (1).

Selenium is an important trace mineral in the body with various properties $(15,16)$. Buntzel et al. and Boucher FR reported that selenium activated the glutathione peroxidase enzyme and had a major role in protection of aerobic tissues

\begin{tabular}{lll}
\hline Table 1. Comparison of the Demographic and Clinical Variables between the Two Groups \\
\hline & Control Group & Selenium Group \\
\hline Age & $58.07 \pm 10.72$ & $58.26 \pm 11.28$ \\
Sex (female) & $(52 \%) 302$ & $(46.5 \%) 81$ \\
Mean predicted risk of CIN & $11.59 \pm 4.12$ & $12.13 \pm 3.98$ \\
Baseline creatinin & $1.01 \pm 0.28$ & $1.04 \pm 0.19$ \\
Diabetes mellitus & $(23 \%) 134$ & $(25 \%) 39$ \\
HTN & $(49 \%) 289$ & $(54 \%) 95$ \\
Smoking & $(26 \%) 154$ & $(25 \%) 38$ \\
Hypercholesterolemia & $(35 \%) 204$ & $(33 \%) 55$ \\
Hematocrit & $39.53 \pm 4.85$ & $41.15 \pm 4.58$ \\
Volume of the used contrast media & $145.22 \pm 24.20$ & $153.40 \pm 35.68$ \\
Incidence of CIN & $22 \%$ & $9 \%$ \\
\hline
\end{tabular}

Abbreviations: CIN, contrast induced nephropathy; HTN, hypertension

\begin{tabular}{lll}
\hline \multicolumn{2}{l}{ Table 2. The Result of Logistic Regression Analysis } & \\
\hline & $\operatorname{Exp}(\mathbf{B})$ & P value \\
\hline Volume of the used contrast media & 0.994 & 0.032 \\
Selenium use & 0.487 & 0.027 \\
Smoking & 1.509 & 0.072 \\
Hyperchlostrolemia & 0.768 & 0.174 \\
Diabetes mellitus & 1.318 & 0.248 \\
HTN & 0.823 & 0.345 \\
Age & 0.992 & 0.349 \\
\hline
\end{tabular}

Abbreviations: CIN, contrast induced nephropathy; HTN, hypertension 
against the injuries caused by free oxygen radicals (17, 18). In the study by Strohmaier et al., selenium reduced renal tubular damage induced by high energy shock wave. It was proposed that free radical scavenging properties of selenium were responsible for this effect (19). Based on the pathogenesis of CIN and evidence regarding the anti-oxidant and free radical scavenging properties of selenium, selenium was proposed as an agent with potential of reducing the incidence of CIN.

Up to now, this has been the first use of selenium in human studies for reducing the incidence of CIN. The key finding of the present study was that prophylactic selenium administration might reduce the incidence of CIN. As in the study by Firouzi et al., we used Mehran's score to match the two groups. Although the hematocrit levels differed between the two groups, the overall risk of nephropathy (based on Mehran's score) was similar and the two groups were comparable (Table 1). According to the results of multivariate analysis, only the volume of the used contrast and use of selenium significantly affected the incidence of CIN. Smoking also had a non-significant effect on increasing the incidence of CIN. The results also revealed that both groups had low baseline risk of CIN (predicted risk of CIN in both groups based on Mehran's algorhythm was about 12, which is a low score). However, the observed incidence of CIN was clearly greater than expected in the control group (22\%). Therefore, some of the differences between two groups regarding the incidence of CIN might have been due to the lower risk profile in the selenium group and not necessarily due to the protective effect of selenium. Also, as mentioned above, the two groups were comparable concerning the predicted risk of CIN, but this study was not randomized and some differences might have been present.

This study had certain limitations. The most important one was that it was not a randomized trial. For the control group, we used the data of another survey performed in the same hospitals. Also, there was no sample size calculation or power analysis. Although both selenium and control groups underwent the same routine hydration regimen before the procedures, due to the non-randomized nature of this study, differences might have been present in the patients' hydration statuses.

Moreover, the patients' baseline selenium or antioxidant status was not determined and selenium supplementation was not adjusted based on these factors. Considering the promising results of the selenium group, selenium supplement can be taken into consideration as a potential candidate for reducing the risk of CIN. Yet, further investigations are required to confirm this finding.

\section{Acknowledgements}

This manuscript was extracted from a student thesis in cardiology approved by Mashhad university of Medical Sciences.

\section{Authors' Contribution}

All authors have had equivalent roles in the preparation of the paper.

\section{Financial disclosure}

There is no financial disclosure.

\section{Funding/Support}

There is no funding/support.

\section{References}

1. McCullough PA. Interface between renal disease and cardiovascular illness. Braunwald's heart disease: a textbook of cardiovascular medicine 7th ed St Louis (MO): WB Saunders. 2005. pp. 2161-72.

2. Firouzi A, Eshraghi A, Shakerian F, Sanati HR, Salehi N, Zahedmehr A, et al. Efficacy of pentoxifylline in prevention of contrast-induced nephropathy in angioplasty patients. International urology and nephrology. 2012;44(4):1145-9.

3. Tublin ME, Murphy ME, Tessler FN. Current concepts in contrast media-induced nephropathy. AJR Am J Roentgenol. 1998;171(4):933-9.

4. Brennan M, Nallamothu B, Patel U. Contrast induced Acute Kidney Injury and the role of Chronic kidney disease in PCI. In: Topol E, Teirstein P, editors. Textbook of interventional cardiology. Canada: 2012. pp. 66-9.

5. Huang SS, Huang PH, Wu TC, Chen JW, Lin SJ. Association of serum bilirubin with contrast-induced nephropathy and future cardiovascular events in patients undergoing coronary intervention. PLoS One. 2012;7(8):e42594.

6. Heyman SN, Rosen S, Khamaisi M, Idee JM, Rosenberger C. Reactive oxygen species and the pathogenesis of radiocontrastinduced nephropathy. Invest Radiol. 2010;45(4):188-95.

7. Staniloae CS, Doucet S, Sharma SK, Katholi RE, Mody KR, Coppola $\mathrm{JT}$, et al. N $\square$ Acetylcysteine Added to Volume Expansion with Sodium Bicarbonate Does Not Further Prevent Contrast $\square$ Induced Nephropathy: Results from the Cardiac Angiography in Renally Impaired Patients Study. Journal of interventional cardiology. 2009;22(3):261-5

8. Amini M, Salarifar M, Amirbaigloo A, Masoudkabir F, Esfahani F. N-acetylcysteine does not prevent contrast-induced nephropathy after cardiac catheterization in patients with diabetes mellitus and chronic kidney disease: a randomized clinical trial. Trials. 2009;10(1):45.

9. Stone GW, McCullough PA, Tumlin JA, Lepor NE, Madyoon $\mathrm{H}$, Murray $\mathrm{P}$, et al. Fenoldopam mesylate for the prevention of contrast-induced nephropathy: a randomized controlled trial. JAMA. 2003;290(17):2284-91.

10. Bügel S, Larsen EH, Sloth JJ, Flytlie K, Overvad K, Steenberg LC, et al. Absorption, excretion, and retention of selenium from a high selenium yeast in men with a high intake of selenium. Food \& nutrition research. 2008;52.

11. Rayman MP. The use of high-selenium yeast to raise selenium status: how does it measure up? British Journal of Nutrition. 2004;92(04):557-73.

12. Levine GN, Bates ER, Blankenship JC, Bailey SR, Bittl JA, Cercek B, et al. 2011 ACCF/AHA/SCAI Guideline for Percutaneous Coronary Intervention: executive summary: a report of the American College of Cardiology Foundation/American Heart Association Task Force on Practice Guidelines and the Society for Cardiovascular Angiography and Interventions. Catheter Cardiovasc Interv. 2012;79(3):453-95.

13. Roozbeh J, Hamidian Jahromi A, Sharifian M, Pakfetrat M, Afshariani R. Protective effect of pentoxifylline on contrast induced nephropathy. Saudi J Kidney Dis Transpl. 2008;19(6):985-6.

14. Yavari V, Ostovan MA, Kojuri J, Afshariani R, Hamidian Jahromi A, Roozbeh J, et al. The preventive effect of pentoxifylline on contrast-induced nephropathy: a randomized clinical trial. Int Urol Nephrol. 2014;46(1):41-6.

15. Ravaglia G, Forti P, Maioli F, Bastagli L, Facchini A, Mariani E, et al. Effect of micronutrient status on natural killer cell immune function in healthy free-living subjects aged $>/=90$ y. Am J Clin Nutr. 2000;71(2):590-8.

16. Zhang ZW, Zhang JL, Gao YH, Wang QH, Li S, Wang XL, et al. Effect of oxygen free radicals and nitric oxide on apoptosis of immune organ induced by selenium deficiency in chickens. Biometals. 2013;26(2):355-65.

17. Boucher FR, Jouan MG, Moro C, Rakotovao AN, Tanguy S, de Leiris J. Does selenium exert cardioprotective effects against oxidative stress in myocardial ischemia? Acta Physiol Hung. 
2008;95(2):187-94.

18. Buntzel J, Riesenbeck D, Glatzel M, Berndt-Skorka R, Riedel $\mathrm{T}$, Mucke R, et al. Limited effects of selenium substitution in the prevention of radiation-associated toxicities. results of a randomized study in head and neck cancer patients. Anticancer
Res. 2010;30(5):1829-32.

19. Strohmaier WL, Lahme S, Weidenbach PM, Bichler KH. Reduction of high-energy shock-wave-induced renal tubular injury by selenium. Urol Res. 1999;27(5):382-5. 